\title{
41
}

\section{Theoretical Description of the Coulomb Interaction by Padé-Jacobi Approximants}

\author{
W. STOLZMANN
}

Institut für Theoretische Physik und Sternuarte der Lniversität Kiel, Olshausenstr. 40, D-24118 Kiel, Germany

\section{T. BLÖCKER}

Institut für Theoretische Physik und Sternwarte der Universität Kiel, Olshausenstr. 40, D-24118 Kiel, Germany

\begin{abstract}
Coulomb interactions for the Free Helmholtz energy and the pressure are studied in a partial new formulation which described more exactly the numerical evaluation of many body theories.
\end{abstract}

\subsection{Introduction}

With regard to the EOS many activities have been developed to yield results which consider different phenomena, for instance pressure dissoziation and ionisation, degeneracy, relativity, Coulomb- and non-Coulombic interactions, pair production and charge mixing in different chemical compositions.

Various theoretical approaches are used in order to include exchange and correlation effects for fully ionized or partially ionized matter (see e.g. Salpeter and Zapolski 1967, Graboske et al. 1969, Hansen 1973, Pokrant 1977, Fontaine et al. 1977, March and Tosi 1984, Perrot and Dharmawardana 1984, Hubbard and Dewitt 1985, Dandrea et al. 1986, van Horn 1987, Kraeft et al. 1986, Ichimaru et al. 1987, Rogers and DeWitt 1987, Däppen et al. 1988, Ichimaru 1990, Eliezer and Ricci 1991, Saumon and Chabrier 1992).

For many applications (e.g. stellar evolution calculations or astroseismology) it is necessary either to have algebraic formulae for the EOS or extensive tables which supply the input informations at any density and temperature. As a first step we present an analytical EOS for fully ionized 
multicomponent plasmas covering a large density-temperature range. The EOS includes non-ideal effects due to exchange-correlation interactions of charged particles at any degeneracy and is applicable to any chemical mixture. Relativistic effects as well as ionic quantum corrections are taken into account. The aim of this contribution is to derive explicite exchange and correlation corrections for the Coulombic part of the EOS in an algebraic form.

Recently, we presented results for the pressure (Stolzmann and Blöcker $1993 \mathrm{a}, \mathrm{b})$ and a first application to the mass-radius relationship of white dwarfs (Stolzmann et al. 1993). This paper will be devoted to a brief overview of the theoretical background of our EOS concept.

\subsection{Theoretical Background}

We start with the Helmholtz free energy $F$ of a fully ionized plasma consisting of $a$ species which is given by

$$
F\left(T, V, N_{a}\right)=\sum_{a} F_{u}^{\mathrm{id}}+F^{\mathrm{coul}}
$$

with $F_{a}^{\text {id }}$ being the ideal free energy and $F^{\text {coul }}$ representing the Coulomb interaction contributions of the free energy. We splitted the Coulomb contributions in the following parts:

$$
F^{\text {coul }}=F_{\mathrm{ee}}^{\mathrm{x}}+F_{\mathrm{ee}}^{\mathrm{c}}+F_{\mathrm{ii}}^{\mathrm{x}}+F_{\mathrm{ii}}^{\mathrm{c}}+F_{\mathrm{ii}}^{\mathrm{cq}}+F_{\mathrm{ie}}^{\mathrm{c}}
$$

where $\mathrm{x}$ and $\mathrm{c}$ denote the exchange and the correlation term, resp. $F_{\mathrm{ee}}$, $F_{\mathrm{ii}}$ and $F_{\mathrm{ie}}$ correspond to the electron-electron, ion-ion and ion-electron interaction. In this notation $F_{\mathrm{ii}}^{\mathrm{cd}}$ and $F_{\mathrm{ii}}^{\mathrm{x}}$ describe ionic quantum corrections.

The pressure is given by the thermodynamic relation

$$
P\left(V, T, N_{u}\right)=-\left(\frac{\partial F}{\partial V}\right)_{T, N}
$$

or equivalent by the grand potential $\Omega$

$$
-\Omega=P \cdot V=G-F
$$

with the definition of the Gibbs free energy

$$
G=\sum_{a} \mu_{a} N_{a} \quad \text { and } \quad \mu_{a}=\left(\frac{\partial F}{\partial N_{a}}\right)_{T, V, N \neq N_{a}}
$$

The equivalence between (3) and (4) is achieved by the elimination of the chemical potential from the pressure (4) by means of an inversion procedure 
described in detail by Kohn and Luttinger (1960), Stolzmann and Kraeft (1979), Perrot and Dharma-wardana (1984), Rösler and Stolzmann (1987). In equivalence to (2) the Coulombic pressure contribution is given by

$$
P^{\text {coul }}=P_{\mathrm{ee}}^{\mathrm{x}}+P_{\mathrm{ee}}^{\mathrm{c}}+P_{\mathrm{ii}}^{\mathrm{x}}+P_{\mathrm{ii}}^{\mathrm{c}}+P_{\mathrm{ii}}^{\mathrm{cq}}+P_{\mathrm{ie}}^{\mathrm{c}}
$$

Consequently, we apply in (2)

$$
\begin{aligned}
F_{\mathrm{e}}^{\mathrm{id}}=\frac{2 k T V}{\Lambda_{\mathrm{e}}^{3}}\{ & \psi\left(J_{1 / 2}(\psi, \lambda)+\frac{3}{2} \lambda J_{3 / 2}(\psi, \lambda)\right) \\
& \left.-\left(J_{3 / 2}(\psi, \lambda)+\frac{5}{2} \lambda J_{5 / 2}(\psi, \lambda)\right)\right\}
\end{aligned}
$$

with the De Broglie wavelength $\Lambda_{\mathrm{e}}$ and the relativity parameter $\lambda$

$$
\Lambda_{\mathrm{e}}=\frac{2 \pi \hbar}{\sqrt{2 m_{\mathrm{e}} \pi k T}} \quad \lambda=\frac{k T}{m c^{2}}
$$

The electron degeneracy parameter $\psi$ is defined implicitly by the density

$$
\begin{aligned}
& n_{\mathrm{e}}=\frac{2}{\Lambda_{\mathrm{e}}^{3}}\left(J_{1 / 2}(\psi, \lambda)+\lambda \frac{3}{2} J_{3 / 2}(\psi, \lambda)\right) \\
& F_{\mathrm{i}}^{\mathrm{id}}=k T V \sum_{j} n_{j}\left\{\ln \left(\frac{n_{j} \Lambda_{j}^{3}}{2}\right)-1\right\}
\end{aligned}
$$

According to Kovetz et al. 1972 we used for the lowest order exchange interaction

$$
\frac{F_{\mathrm{ee}}^{\mathrm{x}}}{N_{\mathrm{e}} k T}=f_{\mathrm{ee}}^{\mathrm{x}}=\frac{\epsilon^{2}}{\pi \Lambda_{\mathrm{e}}^{4}} \frac{1}{n_{\mathrm{e}} k T} J_{\mathrm{rel}}^{\mathrm{x}}(\psi, \lambda) .
$$

with the relativistic Hartree-Fock integral $J_{\text {rel }}^{\mathbf{x}}(\psi, \lambda)$. The non-relativistic conditions can be described by the fit formula of Perrot and Dharmawardana (1984). We determine for the electron-electron correlation term the new Jacobi-Padé approximation

$$
\frac{F_{\mathrm{ee}}^{c}}{N_{\mathrm{e}} k T}=f_{\mathrm{ee}}^{c}=-\frac{a_{0} \Gamma_{\mathrm{e}}^{3 / 2}-a_{2} \Gamma_{\mathrm{e}}^{6} \varepsilon_{\mathrm{c}}\left(r_{\mathrm{s}}, 0\right) / k T}{1+a_{1} \Gamma_{\mathrm{e}}^{3 / 2}+a_{2} \Gamma_{\mathrm{e}}^{6}}
$$

The ground state energy $\varepsilon_{c}$ can be taken from Salpeter and Zalpolsky (1967), Vosko et al. (1980) or Ebeling and Richert (1985). The Coulomb coupling parameter $\Gamma$ describes the ratio of the Coulomb potential to the kinetic 
energy. The Jacobi-Padé approximants of the ion-electron and ion-ion interaction are proposed by Ebeling (1990):

$$
\begin{aligned}
& \frac{F_{\mathrm{ie}}^{\mathrm{c}}}{N_{\mathrm{i}} k T}=f_{\mathrm{ie}}^{\mathrm{c}}=\frac{-c_{0} \Gamma_{\mathrm{i}}^{3 / 2}-c_{2} \Gamma_{\mathrm{e}}^{9 / 2} \varepsilon_{\mathrm{ie}}}{1+\Gamma_{\mathrm{i}}^{3 / 2}\left(c_{1}+2 c_{4} \ln \left[1+\left(c_{5} / \Gamma_{\mathrm{i}}^{3}\right)^{1 / 2}\right]\right)+c_{2} \Gamma_{\mathrm{e}}^{9 / 2}} \\
& \frac{F_{\mathrm{ii}}^{c}}{N_{\mathrm{i}} k T}=f_{\mathrm{ii}}^{\mathrm{c}}=-\frac{b_{0} \Gamma_{\mathrm{i}}^{3 / 2}+b_{2} \Gamma_{\mathrm{i}}^{9 / 2} \varepsilon_{\mathrm{ii}}}{1+b_{1} \Gamma_{\mathrm{i}}^{3 / 2}+b_{2} \Gamma_{\mathrm{i}}^{9 / 2}}
\end{aligned}
$$

The energy $\varepsilon_{\mathrm{ie}}$ is adopted from Ebeling (1990), Hubbard and DeWitt (1985), DeWitt and Hubbard (1976) and Galam and Hansen (1974) whereas $\varepsilon_{\mathrm{ii}}$ consists of Madelung-like and thermal energy and is taken from Stringfellow et al. (1990). Ionic quantum corrections (for the near-classical limit) can be considered according to Chabrier et al. (1992) (see also Nagara et al. 1987):

$$
\begin{aligned}
\frac{F_{\mathrm{ii}}^{\mathrm{cq}}}{N_{\mathrm{i}} k T}=f_{\mathrm{ii}}^{\mathrm{cq}}= & \frac{3}{2} z_{1} \Theta_{\mathrm{i}}+2 \ln \left(1-\mathrm{e}^{-\alpha \Theta_{\mathrm{i}}}\right)+\ln \left(1-\mathrm{e}^{-\gamma \Theta_{\mathrm{i}}}\right) \\
& -3 \ln \Theta_{\mathrm{i}}+2.71848-\Im_{\mathrm{Deb}}\left(\Theta_{\mathrm{i}}\right)
\end{aligned}
$$

with the approximation for the Debye integral $\Im_{\text {Deb }}\left(\Theta_{i}\right)$ :

$$
\Im_{\text {Deb }}\left(\Theta_{i}\right)=\frac{2}{3} \frac{1}{1+0.43 \alpha \Theta_{i}+\frac{5}{\pi^{4}}\left(\alpha \Theta_{i}\right)^{3}}
$$

Leaving the classical region symmetry effects become important. We try to determine the exchange free energy in the lowest order approximation (Hartree-Fock exchange) by

$$
\frac{F_{\mathrm{ii}}^{\mathrm{x}}}{N_{\mathrm{i}} k T}=f_{\mathrm{ii}}^{\mathrm{x}}=-t_{0} \Gamma_{\mathrm{i}} \frac{t_{1}+t_{2} \xi_{\mathrm{i}}^{2}-t_{3} \xi_{\mathrm{i}}^{3}+t_{4} \xi_{\mathrm{i}}^{4}}{1+t_{5} \xi_{\mathrm{i}}^{2}+t_{6} \xi_{\mathrm{i}}^{4}} \tanh \frac{1}{\xi_{\mathrm{i}}}
$$

analogous to the treatment of the electrons by Perrot and Dharma-wardana (1984).

$$
\xi_{\mathrm{i}}=6 \cdot\left(\frac{4}{9 \pi}\right)^{2 / 3} \cdot \frac{\Gamma_{\mathrm{i}}}{\Theta_{\mathrm{i}}^{2}} \quad \Theta_{\mathrm{i}}=\frac{\hbar \omega_{\mathrm{p}}}{k T}
$$

Straightforward we obtain with (3) - (5) for the pressure

$$
\begin{aligned}
& P_{\mathrm{e}}^{\mathrm{id}}=\frac{2 k T}{\Lambda_{\mathrm{e}}^{3}}\left(J_{3 / 2}(\psi, \lambda)+\frac{5}{4} \lambda J_{5 / 2}(\psi, \lambda)\right) \\
& P_{\mathrm{i}}^{\mathrm{id}}=k T \sum_{i} n_{i}
\end{aligned}
$$




$$
\begin{aligned}
& \frac{P_{\mathrm{ee}}^{\mathrm{c}}}{n_{\mathrm{e}} k T}=-\frac{s_{0} \Gamma_{\mathrm{e}}^{3 / 2}-s_{2} \Gamma_{\mathrm{e}}^{6} \mu_{\mathrm{c}}\left(r_{\mathrm{s}}, 0\right) / k T}{1+s_{1} \Gamma_{\mathrm{e}}^{3 / 2}+s_{2} \Gamma_{\mathrm{e}}^{6}}-f_{\mathrm{ee}}^{\mathrm{c}} \\
& \mu_{\mathrm{c}}\left(r_{\mathrm{s}}, 0\right)=\varepsilon_{\mathrm{c}}\left(r_{\mathrm{s}}, 0\right)-\frac{r_{\mathrm{s}}}{3} \frac{\partial \varepsilon_{\mathrm{c}}\left(r_{\mathrm{s}}, 0\right)}{\partial r_{\mathrm{s}}}
\end{aligned}
$$

The pressure of the ion-electron and the ion-ion interaction is given by

$$
\begin{aligned}
\frac{P_{\mathrm{ie}}^{\mathrm{c}}}{n_{\mathrm{i}} k T}= & -D_{\mathrm{ie}} \cdot\left\{\frac{c_{0}}{2} \Gamma_{\mathrm{i}}^{3 / 2}+\left(\frac{3}{2} \varepsilon_{\mathrm{ie}}+\frac{1}{3}\left(h_{\mathrm{ie}}-d_{\mathrm{ie}}\right)\right) c_{2} \Gamma_{\mathrm{e}}^{9 / 2}\right. \\
& \left.+\left(\frac{c_{1}}{2} \Gamma_{\mathrm{i}}^{3 / 2}+c_{4}\left(\ln c_{6}-1+\frac{1}{c_{6}}\right) \Gamma_{\mathrm{i}}^{3 / 2}+\frac{3}{2} c_{2} \Gamma_{\mathrm{e}}^{9 / 2}\right) \cdot f_{\mathrm{ie}}^{\mathrm{c}}\right\} \\
\frac{P_{\mathrm{ii}}^{\mathrm{c}}}{n_{\mathrm{i}} k T}= & -D_{\mathrm{ii}} \Gamma_{\mathrm{i}}^{3 / 2} \cdot\left\{\frac{b_{0}}{2}+\left(\frac{3}{2} \varepsilon_{\mathrm{ii}}+\frac{h_{\mathrm{ii}}}{3}\right) b_{2} \Gamma_{\mathrm{i}}^{3}+\left(\frac{b_{1}}{2}+\frac{3}{2} b_{2} \Gamma_{\mathrm{i}}^{3}\right) f_{\mathrm{ii}}^{\mathrm{c}}\right\}
\end{aligned}
$$

$D_{\mathrm{ie}}$ and $D_{\mathrm{ii}}$ are the denominators of (13) and (14), resp., whereas $h_{\mathrm{kn}}$ and $d_{1 \mathrm{~m}}$ are defined by

$$
h_{\mathrm{lm}}=\Gamma_{\mathrm{i}} \frac{\partial \varepsilon_{\mathrm{lm}}}{\partial \Gamma_{\mathrm{i}}} \quad d_{1 \mathrm{~m}}=r_{\mathrm{s}} \frac{\partial \varepsilon_{\mathrm{lm}}}{\partial r_{\mathrm{s}}} \quad r_{\mathrm{s}}=\left(\frac{3}{4 \pi n_{\mathrm{e}}}\right)^{1 / 3}
$$

Finally, the ionic quantum corrections can be determined by

$$
\begin{aligned}
& \frac{P_{\mathrm{ii}}^{\mathrm{cq}}}{n_{\mathrm{i}} k T}=\frac{3}{4} z_{1} \Theta_{\mathrm{i}}+\frac{1}{2} \gamma \frac{\Theta_{\mathrm{i}}}{\mathrm{e}^{\gamma \Theta_{\mathrm{i}}}-1}+\frac{3}{2} \Im_{\mathrm{Deb}}\left(\Theta_{\mathrm{i}}\right) \\
& \frac{P_{\mathrm{ii}}^{\mathrm{x}}}{n_{\mathrm{i}} k T}=-\Gamma_{\mathrm{i}} q_{0} \frac{1+q_{1} \xi_{\mathrm{i}}^{2}-q_{2} \xi_{\mathrm{i}}^{3}+q_{3} \xi_{\mathrm{i}}^{4}}{1+q_{4} \xi_{\mathrm{i}}^{2}+q_{5} \xi_{\mathrm{i}}^{4}}-f_{\mathrm{ii}}^{\mathrm{x}}
\end{aligned}
$$

A detailed description of our investigations is given by Stolzmann and Blöcker (1993c).

\section{References}

Chabrier, G., Ashcroft, N.W., DeWitt, H.E.: 1992, Nature 360, 48

Däppen, W., Hummer, D. G., Mihalas, D., Weibel-Mihalas, B.: 1988, ApJ. 332, 261

Dandrea, R.D., Ashcroft, N.W., Carlsson, A.E.: 1986, Phys. Rev. B34, 2097

Ebeling, W.: 1990, Contr. Plasma Phys. 30, 553

Ebeling, W., Richert, W.: 1985, phys. stat. sol. (b), 128, 467

Eliezer, S., Ricci, R.A. (eds.): 1991, High Pressure Equation of State: Theory and Applications, Enrico Fermi International School of Physics, Vol. 113

Fontaine, G., Graboske, H. C., van Horn, H. M.: 1977, ApJS 35, 293

Galam, S., Hansen, J.P.: 1976, Phys. Rev. A14, 816 
Graboske, H. C., Harwood Jr., D.J., Rogers, F. J.: 1969, Phys. Rev. 186, 210

Hansen, J. P.: 1973, Phys. Rev. A8, 3096

Hubbard, W. B., DeWit.t, H. E.: 1985, ApJ 290, 388

Ichimaru, S. (ed.): 1990, Strongly Coupled Plasma Physics, North-Holland Delta Series

Ichimaru, S., Iyetomi, H., Tanaka, S.: 1987, Phys. Rep. 149, 91

Kohn, W., Luttinger, M.: 1960, Phys. Rev. 118, 41

Kovetz, A., Lamb, D.Q., van Horn, H.M.: 1972, ApJ. 174, 109

Kraeft, W. D., Kremp, D., Ebeling, W., Röpke, G.: 1986, Quantum statistics of charged particle systems, Plenum, New York

March, N. H., Tosi, M. P.: 1984, Coulomb Liquids, Academic Press, London

Nagara, H., Nagata, Y., Nakamura, T.: 1987, Phys. Rev. A36, 1859

Perrot, F., Dharma-wardana, M. W. C.: 1984, Phys. Rev. A30, 2619

Pokrant, M.A.: 1977, Phys. Rev. A16, 413

Rösler, M., Stolzmann, W.: 1986, phys. stat. sol. (b) 137, 149

Rogers, F.I., DeWitt, H.E. (eds.): 1987, Strongly Coupled Plasma Physics, Plenum Press, New York

Salpeter, E.E., Zapolsky, H.S.: 1967 Phys. Rev. 158, 876

Saumon, D., Chabrier, G.: 1992 Phys. Rev. A46, 2084

Stolzmann, W., Blöcker, T.: 1993a, in Inside the stars, IAU Coll. 137, Astron. Soc. Pac. Conf. Ser. 40, p. 269

Stolzmann, W., Blöcker, T.: 1993b, in White Dwarfs: Advances in Observation and Theory, ed. M. Barstow, NATO ASI Series C, Kluwer, Dordrecht, p. 133

Stolzmann, W., Blöcker, T.: 1993c, A\&A, submitted

Stolzmann, W., Blöcker, T., Rieschick, A.: 1993, in White Duarfs: Advances in Observation and Theory, ed. M. Barstow, NATO ASI Series C, Kluwer, Dordrecht, p. 127

Stolzmann, W., Kraeft, W.D.: 1979, Ann. Phys. 36, 388

Stringfellow, G. S., DeWitt, H. E., Slattery, W. L.: 1990, Phys.Rev. A41, 1105

Van Horn, H.M.: 1987, Mit,t. Astron. Gesellschaft 67,63

Vosko, S.H., Wilk, L., Nusair, M.: 1980, Can. J. Phys. 58, 1200 